\title{
THE $L^{p}$ DIRICHLET PROBLEM FOR ELLIPTIC SYSTEMS ON LIPSCHITZ DOMAINS
}

\author{
ZHONGWEI SHEN
}

May 20, 2005

\begin{abstract}
We develop a new approach to the $L^{p}$ Dirichlet problem via $L^{2}$ estimates and reverse Hölder inequalities. We apply this approach to second order elliptic systems and the polyharmonic equation on a bounded Lipschitz domain $\Omega$ in $\mathbb{R}^{n}$. For $n \geq 4$ and $2-\varepsilon<p<\frac{2(n-1)}{n-3}+\varepsilon$, we establish the solvability of the Dirichlet problem with boundary data in $L^{p}(\partial \Omega)$. In the case of the polyharmonic equation $\Delta^{\ell} u=0$ with $\ell \geq 2$, the range of $p$ is sharp if $4 \leq n \leq 2 \ell+1$.
\end{abstract}

\section{Introduction}

Let $\Omega$ be a bounded Lipschitz domain in $\mathbb{R}^{n}$. Consider the system of second order elliptic operators $(\mathcal{L}(\mathbf{u}))^{r}=-a_{i j}^{r s} D_{i} D_{j} u^{s}$, where $D_{i}=\partial / \partial x_{i}$ and $r, s=$ $1, \ldots, m$. We assume that the coefficients $a_{i j}^{r s}, 1 \leq i, j \leq n, 1 \leq r, s \leq m$ are real constants satisfying the symmetry condition $a_{i j}^{r s}=a_{j i}^{s r}$ and the LegendreHadamard ellipticity condition:

$$
\mu|\xi|^{2}|\eta|^{2} \leq a_{i j}^{r s} \xi_{i} \xi_{j} \eta^{r} \eta^{s} \leq \frac{1}{\mu}|\xi|^{2}|\eta|^{2}
$$

for some $\mu>0$ and any $\xi \in \mathbb{R}^{n}, \eta \in \mathbb{R}^{m}$. The primary purpose of this paper is to study the Dirichlet problem

$$
\left\{\begin{array}{l}
\mathcal{L}(\mathbf{u})=\mathbf{0} \text { in } \Omega \\
\mathbf{u}=\mathbf{f} \in L^{p}(\partial \Omega) \text { on } \partial \Omega \text { and }(\mathbf{u})^{*} \in L^{p}(\partial \Omega),
\end{array}\right.
$$

where $(\mathbf{u})^{*}$ denotes the nontangential maximal function of $\mathbf{u}$, and the boundary value of $\mathbf{u}$ is taken in the sense of nontangential convergence. The following is one of main results of the paper.

2000 Mathematics Subject Classification. 35J55, 35J40.

Key words and phrases. Elliptic Systems; Dirichlet Problems; Polyharmonic Equations; Lipschitz Domains.

Received by the editors May 20, 2005. 
Theorem 1.3. Let $\Omega$ be a bounded Lipschitz domain in $\mathbb{R}^{n}, n \geq 4$ with connected boundary. Then there exists $\varepsilon>0$ depending only on $n, m, \mu$ and $\Omega$ such that, given any $\mathbf{f} \in L^{p}(\partial \Omega)$ with $2-\varepsilon<p<\frac{2(n-1)}{n-3}+\varepsilon$, the Dirichlet problem (1.2) has a unique solution. Moreover, the solution $\mathbf{u}$ satisfies the estimate $\left\|(\mathbf{u})^{*}\right\|_{p} \leq C\|\mathbf{f}\|_{p}$.

With Theorem 1.3 at our disposal, using area integral estimates for elliptic systems [DKPV] and a duality argument found in [V1], we also establish the solvability of the regularity problem with boundary data in $L_{1}^{p}(\partial \Omega)$ for $p$ in the dual range. Here $L_{1}^{p}(\partial \Omega)$ is the space of functions in $L^{p}(\partial \Omega)$ whose first order (tangential) derivatives are also in $L^{p}$.

Theorem 1.4. Let $\Omega$ be a bounded Lipschitz domain in $\mathbb{R}^{n}, n \geq 4$ with connected boundary. Then there exists $\varepsilon_{1}>0$ depending only on $n, m, \mu$ and $\Omega$ such that, given any $\mathbf{f} \in L_{1}^{p}(\partial \Omega)$ with $\frac{2(n-1)}{n+1}-\varepsilon_{1}<p<2+\varepsilon_{1}$, there exists a unique $\mathbf{u}$ satisfying $\mathcal{L}(\mathbf{u})=\mathbf{0}$ in $\Omega, \mathbf{u}=\mathbf{f}$ on $\partial \Omega$, and $(\nabla \mathbf{u})^{*} \in L^{p}(\partial \Omega)$. Moreover, the solution $\mathbf{u}$ may be represented by a single layer potential, and we have $\left\|(\nabla \mathbf{u})^{*}\right\|_{p} \leq C\left\|\nabla_{t} \mathbf{f}\right\|_{p}$, where $\nabla_{t} \mathbf{f}$ denotes the tangential derivatives of $\mathbf{f}$ on $\partial \Omega$.

We remark that for Laplace's equation in Lipschitz domains, the Dirichlet and Neumann problems with boundary data in $L^{p}$ are well understood. Indeed, it has been known since the early 1980's that the Dirichlet problem with optimal estimate $(u)^{*} \in L^{p}$ is uniquely solvable for $2-\varepsilon<p \leq \infty$, and the Neumann problem as well as the regularity problem with optimal estimate $(\nabla u)^{*} \in L^{p}$ is uniquely solvable for $1<p<2+\varepsilon$ (see [D1, D2, JK, V1, DK1, K1]). For elliptic systems as well as higher order elliptic equations, the solvability of the $L^{p}$ boundary value problems were established for $n \geq 3$ and $2-\varepsilon<p<2+\varepsilon$ (see [DKV1, DKV2, FKV, F, V3, G, PV3]). This was achieved by the method of layer potentials. The main tool was certain Rellich-Payne-Weinberg-Necas identities. We mention that for Laplace's equation, the $L^{p}$ estimate $(2<p<\infty)$ for the Dirichlet problem follows directly from the $L^{2}$ estimate and the well known maximum principle, by interpolation. The $L^{p}$ estimate $(1<p<2)$ for the Neumann problem relies on the classical Hölder estimates for solutions of second order elliptic equations of divergence form with bounded measurable coefficients. Similar $L^{\infty}$ and Hölder estimates, however, are not readily available for elliptic systems or higher-order elliptic equations on nonsmooth domains.

Nevertheless, in the case $n=3$, the $L^{p}$ boundary value problems for the optimal ranges of $p$ were solved for elliptic systems [DK2, S1, S2] and higher order elliptic equations [PV1, PV2, PV4] (also see [MM] for systems on manifold). In particular, it was proved that the Dirichlet problem (1.2) is uniquely solvable for $2-\varepsilon<p \leq \infty$. The basic idea in [PV2] is to estimate the decay rate of the Green's function on $\Omega$, using Rellich identities, Caccioppoli inequalities as well as the fact that the $L^{p}$ Dirichlet problem is solvable for some $p<2$. If $n=3$ (or 2), the decay rate obtained is fast enough to yield the Miranda-Agmon maximum principle $\|u\|_{L^{\infty}(\Omega)} \leq C\|u\|_{L^{\infty}(\partial \Omega)}$, from which the $L^{p}$ estimates follows. 
Recently in [S3, S4], we extended this approach to higher dimensions. However, instead of $L^{p}$ estimates, we were only able to establish dilation-invariant estimates in terms of the nontangential maximal functions in the Morrey spaces and their pre-duals, and certain weighted $L^{2}$ estimates with power weights.

In this paper we develop a new approach to the $L^{p}$ Dirichlet problem. The basic idea is as follows. First we prove a reverse Hölder inequality on $\partial \Omega$ :

$$
\left(\frac{1}{\left|\Delta_{r}\right|} \int_{\Delta_{r}}\left|(\mathbf{u})^{*}\right|^{p} d \sigma\right)^{1 / p} \leq C\left(\frac{1}{\left|\Delta_{5 r}\right|} \int_{\Delta_{5 r}}\left|(\mathbf{u})^{*}\right|^{2} d \sigma\right)^{1 / 2},
$$

for $2<p<\frac{2(n-1)}{n-3}+\varepsilon$, where $\Delta_{r}$ is a surface "cube" on $\partial \Omega$, and $\mathbf{u}$ satisfies $\mathcal{L} \mathbf{u}=\mathbf{0}$ in $\Omega,(\mathbf{u})^{*} \in L^{2}(\partial \Omega)$ and $\mathbf{u}=\mathbf{0}$ on $\Delta_{6 r}$. To do this, we use the inequality $(\mathbf{u})^{*} \leq \sup _{K}|\mathbf{u}|+C I_{1}\left((\nabla \mathbf{u})^{*}\right)$, where $K$ is a compact set in $\Omega$ and $I_{1}$ denotes the fractional integral of order one on $\partial \Omega$. The restriction of $p$ comes from the fact that $(\nabla \mathbf{u})^{*}$ is in $L^{q}$ only for some $q>2$. Inequality (1.5) should be considered as a localization estimate in the sense that if the boundary values of two solutions $\mathbf{u}, \mathbf{v}$ agree on $\Delta_{6 r}$, then $(\mathbf{u}-\mathbf{v})^{*}$ behaves far better than either $(\mathbf{u})^{*}$ or $(\mathbf{v})^{*}$ on $\Delta_{r}$.

Let $M_{\Delta_{2 r}}$ denote the Hardy-Littlewood maximal function on $\partial \Omega$, localized to the surface cube $\Delta_{2 r}$. The second step in the proof of Theorem 1.3, which is motivated by a paper of Caffarelli and Peral $[\mathrm{CP}]$, is to establish a good- $\lambda$ type inequality by using a real variable argument and estimate (1.5). Indeed, for $\lambda \geq \lambda_{0}$ and the unique solution of the $L^{2}$ Dirichlet problem $\mathcal{L} \mathbf{u}=\mathbf{0}$ in $\Omega$ and $\mathbf{u}=\mathbf{f}$ on $\partial \Omega$, we will show that

$$
|E(A \lambda)| \leq \delta|E(\lambda)|+\left|\left\{P \in \Delta_{r}: M_{\Delta_{2 r}}\left(|\mathbf{f}|^{2}\right)(P)>\gamma \lambda\right\}\right|,
$$

where $E(\lambda)=\left\{P \in \Delta_{r}: M_{\Delta_{2 r}}\left(\left|(\mathbf{u})^{*}\right|^{2}\right)(P)>\lambda\right\}$, and $A, \delta$ and $\gamma$ are positive constants with the property $\delta A^{p / 2}<1$. The desired estimate $\left\|(\mathbf{u})^{*}\right\|_{p} \leq C\|\mathbf{f}\|_{p}$ follows readily from (1.6) by an integration in $\lambda$.

The proof of estimate (1.6) relies on a Calderón-Zygmund decomposition. The key observation is that if

$$
\frac{1}{\left|\Delta_{12 r}\right|} \int_{\Delta_{12 r}}|\mathbf{u}|^{2} d \sigma \leq \gamma \lambda \text { and } \frac{1}{\left|\Delta_{12 r}\right|} \int_{\Delta_{12 r}}\left|(\mathbf{u})^{*}\right|^{2} d \sigma \leq \lambda
$$

then the localization estimate (1.5) implies that

$$
\left|\left\{P \in \Delta_{r}: M_{\Delta_{2 r}}\left(\left|(\mathbf{u})^{*}\right|^{2}\right)(P)>A \lambda\right\}\right| \leq C\left(\frac{1}{A^{p / 2}}+\frac{\gamma}{A}\right)\left|\Delta_{r}\right| .
$$

At the end of section 2, we use these techniques to formulate a theorem of independent interest on the $L^{p}$ boundedness of operators on $\mathbb{R}^{n}$. It essentially states that if a sublinear operator $T$ is bounded on $L^{2}\left(\mathbb{R}^{n}\right)$, and satisfies a $L^{p}$ 
localization property in the spirit of (1.5) for some $p>2$, then it is bounded on $L^{q}\left(\mathbb{R}^{n}\right)$ for all $2<q<p$ (see Theorem 2.32). This theorem may be considered as a refined version of the well-known Calderón-Zygmund Lemma on weak type $(1,1)$ bounds of $L^{2}$ bounded operators.

We point out that the main ingredients of this new approach are, (1) interior estimates for solutions of $\mathcal{L} \mathbf{u}=0,(2)$ the solvability of the $L^{2}$ Dirichlet problem, (3) the solvability of the regularity problem with data in $L_{1}^{q}(\partial \Omega)$ for some $q>2$. Thus our method also applies to the higher order elliptic equations studied in [DKV1, V3, PV3], where the Dirichlet and regularity problems in $L^{p}$ are solved for $2-\varepsilon<p<2+\varepsilon$. As an example, we consider the Dirichlet problem for the polyharmonic equation:

$$
\left\{\begin{array}{l}
\Delta^{\ell} u=0 \text { in } \Omega \\
D^{\alpha} u=f_{\alpha} \text { for }|\alpha| \leq \ell-2, \frac{\partial^{\ell-1} u}{\partial N^{\ell-1}}=g \text { on } \partial \Omega,\left(\nabla^{\ell-1} u\right)^{*} \in L^{p}(\partial \Omega),
\end{array}\right.
$$

where $\ell \geq 2$. In (1.9), $\alpha=\left(\alpha_{1}, \ldots, \alpha_{n}\right)$ is a multi-index, $D^{\alpha}=D_{1}^{\alpha_{1}} D_{2}^{\alpha_{2}} \cdots D_{n}^{\alpha_{n}}$, and $|\alpha|=\alpha_{1}+\cdots+\alpha_{n}$. Also $N$ denotes the outward unit normal to $\Omega$, and $\frac{\partial^{\ell-1} u}{\partial N^{\ell-1}}=\sum_{|\alpha|=\ell-1} \frac{(\ell-1) !}{\alpha !} N^{\alpha} D^{\alpha} u$.

Theorem 1.10. Let $\Omega$ be a bounded Lipschitz domain in $\mathbb{R}^{n}, n \geq 4$ with connected boundary. Then there exists $\varepsilon>0$ depending on $n, \ell$ and $\Omega$ such that if $f=\left\{f_{\alpha}: 0 \leq|\alpha| \leq \ell-2\right\} \in W A_{\ell-1}^{p}(\partial \Omega)$ and $g \in L^{p}(\partial \Omega)$ with $2-\varepsilon<p<\frac{2(n-1)}{n-3}+\varepsilon$, the Dirichlet problem (1.9) has a unique solution. Moreover, the solution $u$ satisfies

$$
\left\|\left(\nabla^{\ell-1} u\right)^{*}\right\|_{p} \leq C\left\{\|g\|_{p}+\sum_{|\alpha|=\ell-2}\left\|\nabla_{t} f_{\alpha}\right\|_{p}\right\} .
$$

We remark that $W A_{\ell-1}^{p}(\partial \Omega)$ is a Sobolev space of the so-called Whitney arrays on $\partial \Omega$. We refer the reader to [V3] or [PV3] for its definition. As we mentioned earlier, in the case $n=3$, the $L^{p}$ Dirichlet problem (1.9) was solved in $[\mathrm{PV} 1, \mathrm{PV} 2, \mathrm{PV} 4]$ for the optimal range $2-\varepsilon<p \leq \infty$. It was also pointed out in [PV3] that estimate (1.11) fails in general for $p>\frac{2(n-1)}{n-3}$ and $4 \leq n \leq 2 \ell+1$, or $p>\frac{2 \ell}{\ell-1}$ and $n \geq 2 \ell+2$. Thus the range for $p$ in Theorem 1.10 is sharp in the case $4 \leq n \leq 2 \ell+1$.

The paper is organized as follows. Theorem 1.3 is proved in section 2 . The proof of Theorem 1.10, which is very similar to that of Theorem 1.3, is sketched in section 3. Finally we give the proof of Theorem 1.4 in section 4 .

\section{The Dirichlet problem}

Let $\Omega$ be a bounded Lipschitz domain in $\mathbb{R}^{n}$. Assume $0 \in \partial \Omega$ and

$$
\Omega \cap B\left(0, r_{0}\right)=\left\{\left(x^{\prime}, x_{n}\right) \in \mathbb{R}^{n}: x_{n}>\psi\left(x^{\prime}\right)\right\} \cap B\left(0, r_{0}\right),
$$


where $B\left(0, r_{0}\right)$ denotes the ball centered at 0 with radius $r_{0}, \psi: \mathbb{R}^{n-1} \rightarrow \mathbb{R}$ is a Lipschitz function. For $r>0$, we let

$$
\begin{aligned}
& \Delta_{r}=\left\{\left(x^{\prime}, \psi\left(x^{\prime}\right)\right) \in \mathbb{R}^{n}: \quad\left|x_{1}\right|<r, \ldots,\left|x_{n-1}\right|<r\right\}, \\
& D_{r}=\left\{\left(x^{\prime}, x_{n}\right) \in \mathbb{R}^{n}: \quad\left|x_{1}\right|<r, \ldots,\left|x_{n-1}\right|<r, \psi\left(x^{\prime}\right)<x_{n}<\psi\left(x^{\prime}\right)+r\right\} .
\end{aligned}
$$

Note that if $0<r<c r_{0}, \Delta_{r} \subset \partial \Omega$ and $D_{r} \subset \Omega$.

Lemma 2.3. Let $0<r<c r_{0}$. There exists $\varepsilon>0$ depending only on $n, m$, $\mu$ and $\Omega$, such that if $\mathcal{L} \mathbf{u}=\mathbf{0}$ in $\Omega,(\mathbf{u})^{*} \in L^{2}(\partial \Omega)$ and $\mathbf{u}=\mathbf{0}$ on $\Delta_{8 r}$, then $(\mathbf{u})^{*} \in L^{p}\left(\Delta_{r}\right)$ and

$$
\left(\frac{1}{\left|\Delta_{r}\right|} \int_{\Delta_{r}}\left|(\mathbf{u})^{*}\right|^{p} d \sigma\right)^{1 / p} \leq C\left(\frac{1}{\left|\Delta_{5 r}\right|} \int_{\Delta_{5 r}}\left|(\mathbf{u})^{*}\right|^{2} d \sigma\right)^{1 / 2},
$$

where $2<p<2(n-1) /(n-3)+\varepsilon$ and $|\Delta|$ denotes the surface measure of $\Delta$.

Proof. Recall that the nontangential maximal function of $\mathbf{u}$ is defined by

$$
(\mathbf{u})^{*}(P)=\sup \{|\mathbf{u}(x)|: \quad x \in \Omega \text { and } x \in \gamma(P)\} \quad \text { for } P \in \partial \Omega,
$$

where $\gamma(P)=\{x \in \Omega:|x-P|<2 \operatorname{dist}(x, \partial \Omega)\}$. Let

$$
\begin{array}{llll}
\mathcal{M}_{1}(\mathbf{u})(P)=\sup \{|\mathbf{u}(x)|: & x \in \gamma(P) & \text { and } & |x-P| \leq c r\} \\
\mathcal{M}_{2}(\mathbf{u})(P)=\sup \{|\mathbf{u}(x)|: & x \in \gamma(P) & \text { and } & |x-P|>c r\} .
\end{array}
$$

Then $(\mathbf{u})^{*}=\max \left\{\mathcal{M}_{1}(\mathbf{u}), \mathcal{M}_{2}(\mathbf{u})\right\}$. We first estimate $\mathcal{M}_{2}(\mathbf{u})$. To do this, we use interior estimates to obtain

$$
|\mathbf{u}(x)| \leq \frac{C}{r^{n}} \int_{B(x, c r)}|\mathbf{u}(y)| d y \leq \frac{C}{r^{n-1}} \int_{\Delta_{5 r}}\left|(\mathbf{u})^{*}\right| d \sigma
$$

where $x \in \gamma(P), P \in \Delta_{r}$ and $|x-P| \geq c r$. It follows that for any $p>2$,

$$
\begin{aligned}
& \left(\frac{1}{\left|\Delta_{r}\right|} \int_{\Delta_{r}}\left|\mathcal{M}_{2}(\mathbf{u})\right|^{p} d \sigma\right)^{1 / p} \leq \sup _{\Delta_{r}} \mathcal{M}_{2}(\mathbf{u}) \\
& \leq \frac{C}{\left|\Delta_{5 r}\right|} \int_{\Delta_{5 r}}\left|(\mathbf{u})^{*}\right| d \sigma \leq C\left(\frac{1}{\left|\Delta_{5 r}\right|} \int_{\Delta_{5 r}}\left|(\mathbf{u})^{*}\right|^{2} d \sigma\right)^{1 / 2} .
\end{aligned}
$$

Next, to estimate $\mathcal{M}_{1}(\mathbf{u})$ on $\Delta_{r}$, we write

$$
\mathbf{u}\left(x^{\prime}, x_{n}\right)-\mathbf{u}\left(x^{\prime}, \widetilde{x}_{n}\right)=-\int_{x_{n}}^{\widetilde{x}_{n}} \frac{\partial \mathbf{u}}{\partial s}\left(x^{\prime}, s\right) d s
$$


Let $K=\left\{\left(x^{\prime}, x_{n}\right) \in \Omega: \quad\left|x^{\prime}\right|<c r, \psi\left(x^{\prime}\right)+c r<x_{n}<\widetilde{c} r\right\}$. Using (2.9) and interior estimates for $\nabla \mathbf{u}$, it is not hard to show that for $P \in \Delta_{r}$,

$$
\mathcal{M}_{1}(\mathbf{u})(P) \leq \sup _{K}|\mathbf{u}|+C \int_{\Delta_{2 r}} \frac{(\nabla \mathbf{u})_{D_{2 r}}^{*}(Q)}{|P-Q|^{n-2}} d \sigma(Q)
$$

where $(\nabla \mathbf{u})_{D_{2 r}}^{*}$ denotes the nontangential maximal function of $\nabla \mathbf{u}$ with respect to the Lipschitz sub-domain $D_{2 r}$. By the fractional integral estimate, this implies that

$$
\left(\frac{1}{\left|\Delta_{r}\right|} \int_{\Delta_{r}}\left|\mathcal{M}_{1}(\mathbf{u})\right|^{p} d \sigma\right)^{1 / p} \leq \sup _{K}|\mathbf{u}|+C r\left(\frac{1}{\left|\Delta_{2 r}\right|} \int_{\Delta_{2 r}}\left|(\nabla \mathbf{u})_{D_{2 r}}^{*}\right|^{q} d \sigma\right)^{1 / q},
$$

where $\frac{1}{p}=\frac{1}{q}-\frac{1}{n-1}$ and $1<q<n-1$. We now choose $q>2$, depending only on $n, m, \mu$ and $\Omega$, so that the regularity problem for $\mathcal{L} \mathbf{u}=0$ in $D_{\rho r}$ for $2<\rho<3$ with boundary data in $L_{1}^{q}$ is uniquely solvable [G]. It follows that

$$
\int_{\Delta_{2 r}}\left|(\nabla \mathbf{u})_{D_{2 r}}^{*}\right|^{q} d \sigma \leq C \int_{\partial D_{\rho r}}\left|\nabla_{t} \mathbf{u}\right|^{q} d \sigma \leq C \int_{\Omega \cap \partial D_{\rho r}}|\nabla \mathbf{u}|^{q} d \sigma
$$

where we have used the assumption that $\mathbf{u}=0$ on $\Delta_{6 r}$. Integrating both sides of $(2.12)$ in $\rho \in(2,3)$ yields that

$$
\frac{1}{\left|\Delta_{2 r}\right|} \int_{\Delta_{2 r}}\left|(\nabla \mathbf{u})_{D_{2 r}}^{*}\right|^{q} d \sigma \leq \frac{C}{\left|D_{3 r}\right|} \int_{D_{3 r}}|\nabla \mathbf{u}|^{q} d x
$$

By Caccioppoli's inequality as well as its well known consequence, the higher integrability for $\nabla \mathbf{u}[\mathrm{Gi}]$, we obtain

$$
\begin{aligned}
& \left(\frac{1}{\left|D_{3 r}\right|} \int_{D_{3 r}}|\nabla \mathbf{u}|^{q} d x\right)^{1 / q} \leq C\left(\frac{1}{\left|D_{4 r}\right|} \int_{D_{4 r}}|\nabla \mathbf{u}|^{2} d x\right)^{1 / 2} \\
& \leq \frac{C}{r}\left(\frac{1}{\left|D_{5 r}\right|} \int_{D_{5 r}}|\mathbf{u}|^{2} d x\right)^{1 / 2} \leq \frac{C}{r}\left(\frac{1}{\left|\Delta_{5 r}\right|} \int_{\Delta_{5 r}}\left|(\mathbf{u})^{*}\right|^{2} d \sigma\right)^{1 / 2} .
\end{aligned}
$$

Note that $q>2$ implies $p>2(n-1) /(n-3)$. In view of $(2.11),(2.13)$ and (2.14), we have proved that for some $p>2(n-1) /(n-3)$,

$$
\begin{aligned}
\left(\frac{1}{\left|\Delta_{r}\right|} \int_{\Delta_{r}}\left|\mathcal{M}_{1}(\mathbf{u})\right|^{p} d \sigma\right)^{1 / p} & \leq \sup _{K}|\mathbf{u}|+C\left(\frac{1}{\left|\Delta_{5 r}\right|} \int_{\Delta_{5 r}}\left|(\mathbf{u})^{*}\right|^{2} d \sigma\right)^{1 / 2} \\
& \leq C\left(\frac{1}{\left|\Delta_{5 r}\right|} \int_{\Delta_{5 r}}\left|(\mathbf{u})^{*}\right|^{2} d \sigma\right)^{1 / 2} .
\end{aligned}
$$

This, together with (2.8), gives the desired estimate (2.4). 
To prove Theorem 1.3, it will be convenient to work with "cubes" on $\partial \Omega$. Let $D=\left\{\left(x^{\prime}, x_{n}\right) \in \mathbb{R}^{n}: x_{n}>\psi\left(x^{\prime}\right)\right\}$ and $\partial D=\left\{\left(x^{\prime}, \psi\left(x^{\prime}\right)\right): x^{\prime} \in \mathbb{R}^{n-1}\right\}$. Define the map $\Phi: \partial D \rightarrow \mathbb{R}^{n-1}$ by $\Phi\left(x^{\prime}, \psi\left(x^{\prime}\right)\right)=x^{\prime}$. We say that $Q \subset \partial D$ is a cube in $\partial D$, if $\Phi(Q)$ is a cube in $\mathbb{R}^{n-1}$ with sides parallel to the coordinate planes. A cube $\mathrm{Q}$ in $\partial D$ is said to be a dyadic subcube of $Q^{\prime}$ if $\Phi(Q)$ is a dyadic subcube of $\Phi\left(Q^{\prime}\right)$ in $\mathbb{R}^{n-1}$, that is, $\Phi(Q)$ is one of the cubes obtained by bisecting the sides of $\Phi\left(Q^{\prime}\right)$ a finite number of times. Also, if $\Phi(Q)$ is a cube in $\mathbb{R}^{n-1}, \rho \Phi(Q)$ denote the cube which has the same center, but $\rho$ times the side length of $\Phi(Q)$. For a cube $Q$ on $\partial D$, we denote $\Phi^{-1}[\rho \Phi(Q)]$ by $\rho Q$. As an example, in (2.2), $\Delta_{\rho r}=\rho \Delta_{r}$.

For cube $Q$ on $\partial D$ and a function $f$ defined on $Q$, we define a localized Hardy-Littlewood maximal function $M_{Q}$ by

$$
M_{Q}(f)(P)=\sup _{\substack{Q^{\prime} \ni P \\ Q^{\prime} \subset Q}} \frac{1}{\left|Q^{\prime}\right|} \int_{Q^{\prime}}|f| d \sigma .
$$

In the next lemma, we will estimate $|E(\lambda)|$, where

$$
E(\lambda)=\left\{P \in \Delta_{r}: M_{\Delta_{2 r}}\left(\left|(\mathbf{u})^{*}\right|^{2}\right)(P)>\lambda\right\}, \quad 0<r<c r_{0} .
$$

Lemma 2.18. Suppose that $2<p<\frac{2(n-1)}{n-3}+\varepsilon$, where $\varepsilon>0$ is the same as in Lemma 2.3. There exist positive constants $A, \delta, \gamma$ and $C_{0}$ depending only on $n$, $m, \mu, p$ and $\Omega$ such that if $\mathcal{L} \mathbf{u}=\mathbf{0}$ in $\Omega,(\mathbf{u})^{*} \in L^{2}(\partial \Omega)$ and $\mathbf{u}=\mathbf{f} \in L^{2}(\partial \Omega)$ on $\partial \Omega$, then

$$
|E(A \lambda)| \leq \delta|E(\lambda)|+\left|\left\{P \in \Delta_{r}: M_{\Delta_{2 r}}\left(|\mathbf{f}|^{2}\right)(P)>\gamma \lambda\right\}\right|
$$

for all $\lambda \geq \lambda_{0}$, where

$$
\lambda_{0}=\frac{C_{0}}{\left|\Delta_{2 r}\right|} \int_{\Delta_{2 r}}\left|(\mathbf{u})^{*}\right|^{2} d \sigma
$$

Most importantly, the constants $A, \delta$ satisfy the condition $\delta A^{p / 2}<1$.

Proof. We use a real variable argument which is motivated by the method of approximation in $[\mathrm{CP}]$. We begin by fixing $p$ so that $2<p<2(n-1) /(n-3)+\varepsilon$. Let $\delta \in(0,1)$ be a small constant to be determined. By the weak $(1,1)$ estimate of the Hardy-Littlewood maximal function,

$$
|E(\lambda)| \leq \frac{C\left(n,\|\psi\|_{\infty}\right)}{\lambda} \int_{\Delta_{2 r}}\left|(\mathbf{u})^{*}\right|^{2} d \sigma
$$

Thus, if $\lambda \geq \lambda_{0}$ where $\lambda_{0}$ is given in (2.20) with a large $C_{0}$, we have $|E(\lambda)|<$ $\delta\left|\Delta_{r}\right|$. Let $A=1 /\left(2 \delta^{2 / p}\right)$. Note that $\delta A^{p / 2}=1 / 2^{p / 2}<1 / 2$. 
Since $E(\lambda)$ is open relative to $\Delta_{r}$, there exists a collection of disjoint dyadic subcubes $\left\{Q_{k}\right\}$ of $\Delta_{r}$ such that $E(\lambda)=\bigcup_{k} Q_{k}$. Clearly we may assume that each $Q_{k}$ is maximal in the sense that $\widetilde{Q}_{k}$ is not contained in $E(\lambda)$, where $\widetilde{Q}_{k}$ denotes the dyadic "parent" of $Q_{k}$, i.e., $Q_{k}$ is one of $2^{n-1}$ cubes obtained by subdividing $\widetilde{Q}_{k}$ once. Since $|E(\lambda)|<\delta\left|\Delta_{r}\right|$, we may also assume that $32 Q_{k} \subset \Delta_{2 r}$ by taking $\delta$ sufficient small.

We claim that it is possible to choose positive constants $\delta, \gamma$ and $C_{0}$ so that if $\left\{P \in Q_{k}: M_{\Delta_{2 r}}\left(|\mathbf{f}|^{2}\right)(P) \leq \gamma \lambda\right\} \neq \emptyset$, then

$$
\left|E(A \lambda) \cap Q_{k}\right| \leq \delta\left|Q_{k}\right|
$$

Estimate (2.19) follows from (2.22) by summation.

It remains to prove the claim. Suppose that $\left\{P \in Q_{k}: M_{\Delta_{2 r}}\left(|\mathbf{f}|^{2}\right)(P) \leq\right.$ $\gamma \lambda\} \neq \emptyset$. Since $Q_{k}$ is maximal, by a simple geometric observation, we obtain

$$
M_{\Delta_{2 r}}\left(\left|(\mathbf{u})^{*}\right|^{2}\right)(P) \leq \max \left(M_{2 Q_{k}}\left(\left|(\mathbf{u})^{*}\right|^{2}\right)(P), C_{1} \lambda\right)
$$

for any $P \in Q_{k}$, where $C_{1}$ depends only on $n$ and $\|\nabla \psi\|_{\infty}$. We may assume that $A \geq C_{1}$ by making $\delta$ small. It follows that

$$
\left|Q_{k} \cap E(A \lambda)\right| \leq\left|\left\{P \in Q_{k}: M_{2 Q_{k}}\left(\left|(\mathbf{u})^{*}\right|^{2}\right)(P)>A \lambda\right\}\right| .
$$

Also note that, if $\widetilde{Q}_{k} \subset Q \subset \Delta_{2 r}$, then

$$
\frac{1}{|Q|} \int_{Q}|\mathbf{f}|^{2} d \sigma \leq \gamma \lambda \text { and } \frac{1}{|Q|} \int_{Q}\left|(\mathbf{u})^{*}\right|^{2} d \sigma \leq \lambda
$$

Let $\mathbf{v}=\mathbf{v}_{k}$ be the unique solution of the $L^{2}$ Dirichlet problem on $\Omega$ with boundary data $\mathbf{f} \chi_{16 Q_{k}}$. It follows from (2.24) and Lemma 2.3 that

$$
\begin{aligned}
& \left|Q_{k} \cap E(A \lambda)\right| \leq\left|\left\{P \in Q_{k}: M_{2 Q_{k}}\left(\left|(\mathbf{u}-\mathbf{v})^{*}\right|^{2}\right)(P)>\frac{A \lambda}{4}\right\}\right| \\
& +\left|\left\{P \in Q_{k}: M_{2 Q_{k}}\left(\left|(\mathbf{v})^{*}\right|^{2}\right)(P)>\frac{A \lambda}{4}\right\}\right| \\
& \leq \frac{C}{(A \lambda)^{\bar{p} / 2}} \int_{2 Q_{k}}\left|(\mathbf{u}-\mathbf{v})^{*}\right|^{\bar{p}} d \sigma+\frac{C}{A \lambda} \int_{2 Q_{k}}\left|(\mathbf{v})^{*}\right|^{2} d \sigma \\
& \leq\left|Q_{k}\right|\left\{C\left(\frac{1}{A \lambda\left|10 Q_{k}\right|} \int_{10 Q_{k}}\left|(\mathbf{u}-\mathbf{v})^{*}\right|^{2} d \sigma\right)^{\bar{p} / 2}+\frac{C}{A \lambda\left|16 Q_{k}\right|} \int_{16 Q_{k}}|\mathbf{f}|^{2} d \sigma\right\},
\end{aligned}
$$

where $p<\bar{p}<2(n-1) /(n-3)+\varepsilon$ and we also used the $L^{2}$ estimate for $\mathbf{v}$ in the last inequality. This, together with (2.25), the $L^{2}$ estimate and the choice $A=1 /(2 \delta)^{2 / p}$, gives

$$
\left|Q_{k} \cap E(A \lambda)\right| \leq\left|Q_{k}\right|\left\{\frac{C_{2}}{A^{\bar{p} / 2}}+\frac{C_{2} \gamma}{A}\right\}=\delta\left|Q_{k}\right|\left\{C_{2} 2^{\bar{p} / 2} \delta^{\frac{\bar{p}}{p}-1}+2 C_{2} \gamma \delta^{\frac{2}{p}-1}\right\},
$$


where $C_{2}$ depends only on $n, m, \mu, \bar{p}$ and $\Omega$.

Finally, since $\bar{p}>p$, we may choose $\delta>0$ so small that $C_{2} 2^{\bar{p} / 2} \delta^{\frac{\bar{p}}{p}-1}<1 / 2$. With $\delta$ fixed, we choose $\gamma>0$ so small that $2 C_{2} \gamma \delta^{\frac{2}{p}-1}<1 / 2$. We then obtain $\left|Q_{k} \cap E(A \lambda)\right|<\delta\left|Q_{k}\right|$. The proof is complete.

Estimate (2.19) is a good- $\lambda$ type inequality. Its relation with the $L^{p}$ norm estimate is well known.

Lemma 2.27. Suppose that $2<p<\frac{2(n-1)}{n-3}+\varepsilon$ where $\varepsilon>0$ is the same as in Lemma 2.3. If $\mathbf{f} \in L^{p}(\partial \Omega)$ and $\mathbf{u}$ is the unique solution of the $L^{2}$ Dirichlet problem with boundary data $\mathbf{f}$, then $(\mathbf{u})^{*} \in L^{p}\left(\Delta_{r}\right)$ and

$$
\begin{aligned}
& \left(\frac{1}{\left|\Delta_{r}\right|} \int_{\Delta_{r}}\left|(\mathbf{u})^{*}\right|^{p} d \sigma\right)^{1 / p} \\
& \leq C\left(\frac{1}{\left|\Delta_{2 r}\right|} \int_{\Delta_{2 r}}\left|(\mathbf{u})^{*}\right|^{2} d \sigma\right)^{1 / 2}+C\left(\frac{1}{\left|\Delta_{2 r}\right|} \int_{\Delta_{2 r}}|\mathbf{f}|^{p} d \sigma\right)^{1 / p} .
\end{aligned}
$$

Proof. We begin by multiplying both sides of (2.19) by $\lambda^{\frac{p}{2}-1}$ and integrating the resulting inequality in $\lambda$ on the interval $\left(\lambda_{0}, \Lambda\right)$. This gives

$$
\frac{1}{A^{p / 2}} \int_{A \lambda_{0}}^{A \Lambda} \lambda^{\frac{p}{2}-1}|E(\lambda)| d \lambda \leq \delta \int_{\lambda_{0}}^{\Lambda} \lambda^{\frac{p}{2}-1}|E(\lambda)| d \lambda+C \int_{\Delta_{2 r}}|\mathbf{f}|^{p} d \sigma .
$$

It follows that

$$
\begin{aligned}
\left(\frac{1}{A^{p / 2}}-\delta\right) \int_{0}^{\Lambda} \lambda^{\frac{p}{2}-1}|E(\lambda)| d \lambda & \leq C \int_{0}^{A \lambda_{0}} \lambda^{\frac{p}{2}-1}|E(\lambda)| d \lambda+C \int_{\Delta_{2 r}}|\mathbf{f}|^{p} d \sigma \\
& \leq C \lambda_{0}^{\frac{p}{2}}\left|\Delta_{2 r}\right|+C \int_{\Delta_{2 r}}|\mathbf{f}|^{p} d \sigma
\end{aligned}
$$

Since $\delta A^{p / 2}<1$, estimate (2.28) follows easily from the above inequality and (2.20) by letting $\Lambda \rightarrow \infty$.

We are now in a position to give the proof of Theorem 1.3.

Proof of Theorem 1.3. The case $2-\varepsilon<p \leq 2$ is already known [G]. For $p>2$, since $L^{p}(\partial \Omega) \subset L^{2}(\partial \Omega)$ for $p>2$, the uniqueness follows from the uniqueness for the Dirichlet problem with $L^{2}$ data. The existence as well as the estimate $\left\|(\mathbf{u})^{*}\right\|_{p} \leq C\|\mathbf{f}\|_{p}$ follows easily from Lemma 2.27 by covering $\partial \Omega$ with a finite number of coordinate patches.

Remark 2.30. Theorem 1.3 also holds for the exterior domain $\Omega_{-}=\mathbb{R}^{n} \backslash \bar{\Omega}$. That is, given any $\mathbf{f} \in L^{p}(\partial \Omega)$ with $2 \leq p<2(n-1) /(n-3)+\varepsilon$, there exists a unique $\mathbf{u}$ on $\Omega_{-}$satisfying $\mathcal{L} \mathbf{u}=\mathbf{0}$ in $\Omega_{-}, \mathbf{u}=\mathbf{f}$ on $\partial \Omega,(\mathbf{u})_{e}^{*} \in L^{p}(\partial \Omega)$, and $|\mathbf{u}(x)|=O\left(|x|^{2-n}\right)$ as $|x| \rightarrow \infty$. Moreover, the solution $\mathbf{u}$ satisfies $\left\|(\mathbf{u})_{e}^{*}\right\|_{p} \leq$ 
$C\|\mathbf{f}\|_{p}$, where $(\mathbf{u})_{e}^{*}$ denotes the nontangential maximal function of $\mathbf{u}$ with respect to $\Omega_{-}$.

Remark 2.31. In the case $n=3$, the techniques in this section yield the $L^{p}$ solvability of the Dirichlet problem for $2<p<\infty$. To see this, we note that estimate (2.11) holds for any $1<p \leq \infty$, if $q>n-1$.

We end this section by formulating a theorem, which is of independent interest, on the $L^{p}$ boundedness of operators on $\mathbb{R}^{n}$. Its proof, which is given in [S5], may be carried out by slightly modifying the techniques used in the proof of Lemma 2.18. We point out that any operator with a standard CalderónZygmund kernel satisfies condition (2.33) for all $p>2$.

Theorem 2.32. Let $T$ be a bounded sublinear operator on $L^{2}\left(\mathbb{R}^{n}\right)$. Suppose that for some $p>2$, $T$ satisfies the following $L^{p}$ localization property:

$$
\begin{aligned}
& \left\{\frac{1}{|B|} \int_{B}|T f|^{p} d x\right\}^{1 / p} \\
& \leq C\left\{\left(\frac{1}{|2 B|} \int_{2 B}|T f|^{2} d x\right)^{1 / 2}+\sup _{B^{\prime} \supset B}\left(\frac{1}{\left|B^{\prime}\right|} \int_{B^{\prime}}|f|^{2} d x\right)^{1 / 2}\right\},
\end{aligned}
$$

for any ball $B \subset \mathbb{R}^{n}$ and any $C^{\infty}$ function $f$ with $\operatorname{supp}(f) \subset \mathbb{R}^{n} \backslash 3 B$. Then $T$ is bounded on $L^{q}\left(\mathbb{R}^{n}\right)$ for any $2<q<p$.

\section{The Polyharmonic equation}

In this section we give the proof of Theorem 1.10, using the same line of argument as in the last section. We will need the following estimates for suitable solutions of $\Delta^{\ell} u=0$ on $\Omega$ :

$$
\begin{aligned}
\left\|\left(\nabla^{\ell-1} u\right)^{*}\right\|_{2} & \leq C\left\|\nabla^{\ell-1} u\right\|_{2}, \\
\left\|\left(\nabla^{\ell} u\right)^{*}\right\|_{q} & \leq C\left\|\nabla_{t} \nabla^{\ell-1} u\right\|_{q},
\end{aligned}
$$

for some $q>2$ depending only on $n, \ell$, and the Lipschitz character of $\Omega$. Both estimates were established in [V3] for any integer $\ell \geq 2$. In the case of the biharmonic equation $(\ell=2)$, estimate (3.1) was obtained earlier in [DKV1]. We also need a Caccioppoli's inequality,

$$
\int_{D_{s r}}\left|\nabla^{\ell} u\right|^{2} d x \leq \frac{C}{(\rho-s)^{2} r^{2}} \int_{D_{\rho r}}\left|\nabla^{\ell-1} u\right|^{2} d x
$$

where $0<s<\rho<1, u$ satisfies $\Delta^{\ell} u=0$ in $\Omega$, $\left(\nabla^{\ell} u\right)^{*} \in L^{2}\left(\Delta_{2 r}\right)$, and $D^{\alpha} u=0$ on $\Delta_{3 r}$ for all $0 \leq|\alpha| \leq \ell-1$. Inequality (3.3) may be proved by using integration by parts and Poincaré's inequalities for functions which vanish on part of the boundary. From (3.3) one may deduce the following boundary reverse Hölder inequality for some $q>2$ by a standard argument,

$$
\left(\frac{1}{\left|D_{r}\right|} \int_{D_{r}}\left|\nabla^{\ell} u\right|^{q} d x\right)^{1 / q} \leq C\left(\frac{1}{\left|D_{2 r}\right|} \int_{D_{2 r}}\left|\nabla^{\ell} u\right|^{2} d x\right)^{1 / 2}
$$


Lemma 3.5. There exists $\varepsilon>0$ depending only on $n, \ell$ and $\Omega$ such that if $\Delta^{\ell} u=0$ in $\Omega,\left(\nabla^{\ell-1} u\right)^{*} \in L^{2}(\partial \Omega)$ and $D^{\alpha} u=0$ for all $0 \leq|\alpha| \leq \ell-1$ on the surface cube $\Delta_{8 r}$, then $\left(\nabla^{\ell-1} u\right)^{*} \in L^{p}\left(\Delta_{r}\right)$ and

$$
\left(\frac{1}{\left|\Delta_{r}\right|} \int_{\Delta_{r}}\left|\left(\nabla^{\ell-1} u\right)^{*}\right|^{p} d \sigma\right)^{1 / p} \leq C\left(\frac{1}{\left|\Delta_{5 r}\right|} \int_{\Delta_{5 r}}\left|\left(\nabla^{\ell-1} u\right)^{*}\right|^{2} d \sigma\right)^{1 / 2}
$$

where $2<p<\frac{2(n-1)}{n-3}+\varepsilon$.

Proof. Using the well known interior estimates, one may prove that for $P \in \Delta_{r}$,

$$
\begin{aligned}
& \left(\nabla^{\ell-1} u\right)^{*}(P) \\
& \leq C\left(\frac{1}{\left|\Delta_{5 r}\right|} \int_{\Delta_{5 r}}\left|\left(\nabla^{\ell-1} u\right)^{*}\right|^{2} d \sigma\right)^{1 / 2}+C \int_{\Delta_{2 r}} \frac{\left(\nabla^{\ell} u\right)_{D_{2 r}}^{*}(Q)}{|P-Q|^{n-2}} d \sigma(Q) .
\end{aligned}
$$

With estimates (3.2), (3.4) and (3.3) at our disposal, the rest of the proof is similar to that of Lemma 2.1. We omit the details.

As in (2.17), we let

$$
F(\lambda)=\left\{P \in \Delta_{r}: M_{\Delta_{2 r}}\left(\left|\left(\nabla^{\ell-1} u\right)^{*}\right|^{2}\right)(P)>\lambda\right\} \quad \text { for } 0<r<c r_{0} .
$$

Lemma 3.8. Suppose that $2<p<\frac{2(n-1)}{n-3}+\varepsilon$ where $\varepsilon>0$ is the same as in Lemma 3.5. There exist positive constants $A, \delta, \gamma$ and $C_{0}$ depending only on $n$, $\ell, p$ and $\Omega$, such that if $f=\left\{f_{\alpha}: 0 \leq|\alpha| \leq \ell-2\right\} \in W A_{\ell-1}^{2}(\partial \Omega), g \in L^{2}(\partial \Omega)$ and $u$ is the unique solution of the $L^{2}$ Dirichlet problem (1.9) with boundary data $f, g$, then

$$
|F(A \lambda)| \leq \delta|F(\lambda)|+\left|\left\{P \in \Delta_{r}: M_{\Delta_{2 r}}\left\{|g|^{2}+\sum_{|\alpha|=\ell-2}\left|\nabla_{t} f_{\alpha}\right|^{2}\right\}(P)>\gamma \lambda\right\}\right|
$$

for all $\lambda \geq \lambda_{0}$, where

$$
\lambda_{0}=\frac{C_{0}}{\left|\Delta_{2 r}\right|} \int_{\Delta_{2 r}}\left|\left(\nabla^{\ell-1} u\right)^{*}\right|^{2} d \sigma
$$

Most importantly, the constants $A, \delta$ satisfy the condition $\delta A^{p / 2}<1$.

Proof. The proof is similar to that of Lemma 2.18. However, in the place of $\mathbf{v}$, we have to be more careful with the choice of polyharmonic function $v=v_{k}$ in $\Omega$ for each $Q_{k}$, since $W A_{\ell-1}^{2}(\partial \Omega)$ is a Sobolev space. Let $\varphi=\varphi_{k}$ is a smooth cut-off function on $\mathbb{R}^{n}$ such that $\varphi=1$ on $16 Q_{k}, \varphi=0$ on $\partial \Omega \backslash 17 Q_{k},\left|D^{\alpha} \varphi\right| \leq C / r^{|\alpha|}$ for $0 \leq|\alpha| \leq \ell-1$, where $r=r_{k}$ is the diameter of $Q_{k}$. Let $h$ be a polynomial 
of degree $\ell-2$ to be determined. We choose $v=v_{k}$ to be the solution of $L^{2}$ Dirichlet problem (1.9) with boundary data

$$
D^{\alpha} v=D^{\alpha}((u-h) \varphi)=\sum_{0 \leq \beta \leq \alpha} \frac{\alpha !}{\beta !(\alpha-\beta) !}\left(f_{\beta}-D^{\beta} h\right) D^{\alpha-\beta} \varphi
$$

for $0 \leq|\alpha| \leq \ell-2$ and $\frac{\partial^{\ell-1} v}{\partial N^{\ell-1}}=g \varphi$ on $\partial \Omega$.

Write $u=v+w+h$. Then $\nabla^{\ell-1} u=\nabla^{\ell-1} v+\nabla^{\ell-1} w$, since $h$ is a polynomial of degree $\ell-2$. It is easy to see that $w$ is the solution of the $L^{2}$ Dirichlet problem (1.9) with boundary values vanishing on $16 Q_{k}$. In fact, $D^{\alpha} w=D^{\alpha}((u-h)(1-$ $\varphi)$ ) for $0 \leq|\alpha| \leq \ell-2$ and $\frac{\partial^{\ell-1} w}{\partial N^{\ell-1}}=g(1-\varphi)$ on $\partial \Omega$. Thus we may apply Lemma 3.5 to $w$.

To control the $L^{2}$ norm of $\left(\nabla^{\ell-1} v\right)^{*}$, we note that for $|\alpha|=\ell-2$

$$
\begin{aligned}
& \left\|\nabla_{t} \nabla^{\alpha}((u-h) \varphi)\right\|_{2} \\
& \leq C \sum_{0 \leq \beta \leq \alpha}\left\{\left\|\left(\nabla_{t}\left(f_{\beta}-D^{\beta} h\right)\right) D^{\alpha-\beta} \varphi\right\|_{2}+\left\|\left(f_{\beta}-D^{\beta} h\right) \nabla_{t} D^{\alpha-\beta} \varphi\right\|_{2}\right\} \\
& \leq C \sum_{0 \leq \beta \leq \alpha} r^{|\beta|-\ell+2}\left\{\left\|\nabla_{t}\left(f_{\beta}-D^{\beta} h\right)\right\|_{L^{2}\left(17 Q_{k}\right)}+r^{-1}\left\|f_{\beta}-D^{\beta} h\right\|_{L^{2}\left(17 Q_{k}\right)}\right\} .
\end{aligned}
$$

Finally, we let $h(x)=\sum_{|\alpha|<\ell-2} \frac{B_{\alpha}}{\alpha !} x^{\alpha}$, where the constants $B_{\alpha}$ are defined inductively by

$$
\begin{aligned}
B_{\alpha} & =\frac{1}{\left|17 Q_{k}\right|} \int_{17 Q_{k}} f_{\alpha}(P) d \sigma(P) \text { for }|\alpha|=\ell-2, \\
B_{\alpha} & =\frac{1}{\left|17 Q_{k}\right|} \int_{17 Q_{k}}\left\{f_{\alpha}(P)-\sum_{\substack{|\alpha| \leq \ell-2 \\
\alpha>\beta}} \frac{B_{\alpha}}{(\alpha-\beta) !} P^{\alpha-\beta}\right\} d \sigma(P)
\end{aligned}
$$

for $0 \leq|\alpha|<\ell-2$. We remark that $B_{\alpha}$ is defined in such a way that

$$
\int_{17 Q_{k}}\left\{f_{\beta}-D^{\beta} h\right\} d \sigma=0 \text { for all } 0 \leq|\beta| \leq \ell-2 .
$$

From this, by using Poincaré's inequality repeatedly, we obtain

$$
\begin{aligned}
\left\|f_{\beta}-D^{\beta} h\right\|_{L^{2}\left(17 Q_{k}\right)} & \leq C r \sum_{|\alpha|=|\beta|+1}\left\|f_{\alpha}-D^{\alpha} h\right\|_{L^{2}\left(17 Q_{k}\right)} \\
& \leq C r^{\ell-|\beta|-2} \sum_{|\alpha|=\ell-2}\left\|f_{\alpha}-D^{\alpha} h\right\|_{L^{2}\left(17 Q_{k}\right)} \\
& \leq C r^{\ell-|\beta|-1} \sum_{|\alpha|=\ell-2}\left\|\nabla_{t} f_{\alpha}\right\|_{L^{2}\left(17 Q_{k}\right)} .
\end{aligned}
$$


By combining estimates (3.12) with (3.15), we get

$$
\left\|\left(\nabla^{\ell-1} v\right)^{*}\right\|_{2} \leq C\left\{\|g\|_{L^{2}\left(17 Q_{k}\right)}+\sum_{|\alpha|=\ell-2}\left\|\nabla_{t} f_{\alpha}\right\|_{L^{2}\left(17 Q_{k}\right)}\right\} .
$$

With these observations, the argument in Lemma 2.18 goes through with minor changes. We leave the details to the reader.

The following lemma follows from Lemma 3.4 by integration.

Lemma 3.17. Suppose that $2<p<\frac{2(n-1)}{n-3}+\varepsilon$, where $\varepsilon>0$ is the same as in Lemma 3.5. If $f \in W A_{\ell-1}^{p}(\partial \Omega), g \in L^{p}(\partial \Omega)$, and $u$ is the polyharmonic function on $\Omega$ satisfying $\left(\nabla^{\ell-1} u\right)^{*} \in L^{2}(\partial \Omega)$ and $D^{\alpha} u=f_{\alpha}$ for $0 \leq|\alpha| \leq \ell-2$, $\frac{\partial^{\ell-1} u}{\partial N^{\ell-1}}=g$ on $\partial \Omega$, then $\left(\nabla^{\ell-1} u\right)^{*} \in L^{p}\left(\Delta_{r}\right)$ and

$$
\begin{gathered}
\left\{\frac{1}{\left|\Delta_{r}\right|} \int_{\Delta_{r}}\left|\left(\nabla^{\ell-1} u\right)^{*}\right|^{p} d \sigma\right\}^{1 / p} \leq C\left\{\frac{1}{\left|\Delta_{2 r}\right|} \int_{\Delta_{2 r}}\left|\left(\nabla^{\ell-1} u\right)^{*}\right|^{2} d \sigma\right\}^{1 / 2} \\
+C\left\{\frac{1}{\left|\Delta_{2 r}\right|} \int_{\Delta_{2 r}}\left(|g|^{p}+\sum_{|\alpha|=\ell-2}\left|\nabla_{t} f_{\alpha}\right|^{p}\right) d \sigma\right\}^{1 / p} .
\end{gathered}
$$

As before, Theorem 1.10 is a consequence of the $L^{2}$ solvability and Lemma 3.17 .

\section{The regularity problem for elliptic systems}

This section is devoted to the proof of Theorem 1.4. We follow an approach found in [V1], where it was used by Verchota to establish the solvability of the $L^{p}$ regularity problem for Laplace's equation. The basic idea is to prove

$$
\left\|\frac{\partial \mathbf{u}}{\partial \nu}\right\|_{p} \leq C\left\|\nabla_{t} \mathbf{u}\right\|_{p}
$$

for starshaped Lipschitz domains, using a duality argument which involves conjugate functions and area integral estimates. With (4.1) one may show that the single layer potential, which maps $L^{p}(\partial \Omega)$ to $L_{1}^{p}(\partial \Omega)$, is invertible. We should mention that in (4.1), $\frac{\partial \mathbf{u}}{\partial \nu}$ denotes the conormal derivative defined by $\left(\frac{\partial \mathbf{u}}{\partial \nu}\right)^{r}=a_{i j}^{r s} \frac{\partial u^{s}}{\partial x_{j}} N_{i}$.

Lemma 4.2. Let $\Omega$ be a starshaped Lipschitz domain in $\mathbb{R}^{n}, n \geq 3$. Suppose that for some $p>1$, the $L^{p}$ Dirichlet problem (1.2) is uniquely solvable for any $\mathbf{f} \in L^{p}(\partial \Omega)$. If $\mathcal{L} \mathbf{u}=\mathbf{0}$ in $\Omega,(\nabla \mathbf{u})^{*} \in L^{p^{\prime}}(\partial \Omega)$ and $\nabla \mathbf{u}$ has nontangential limit on $\partial \Omega$, then $\left\|\frac{\partial \mathbf{u}}{\partial \nu}\right\|_{p^{\prime}} \leq C\left\|\nabla_{t} \mathbf{u}\right\|_{p^{\prime}}$ where $p^{\prime}=p /(p-1)$.

Proof. We may assume that $\Omega$ is starshaped with respect to the origin. Let $\mathbf{g}$ be a Lipschitz continuous function on $\partial \Omega$ and $\mathbf{v}$ be the solution of $\mathcal{L} \mathbf{v}=0$ in $\Omega$ satisfying $(\nabla \mathbf{v})^{*} \in L^{2}(\partial \Omega)$ and $\mathbf{v}=\mathbf{g}$ on $\partial \Omega$. Define

$$
\mathbf{H}(x)=\int_{0}^{1} \mathbf{w}(r x) \frac{d r}{r} \quad \text { for } x \in \Omega,
$$


where $\mathbf{w}(x)=\mathbf{v}(x)-\mathbf{v}(0)$. It is easy to verify that $\mathcal{L} \mathbf{H}=\mathbf{0}$ and $\frac{\partial H^{r}}{\partial x_{i}} x_{i}=w^{r}$. As in the case of harmonic functions, we also have

$$
\left\|(\nabla \mathbf{H})^{*}\right\|_{p} \leq C\left\|(\mathbf{w})^{*}\right\|_{p} \leq C\|\mathbf{g}\|_{p} .
$$

The first inequality in (4.4), which holds for any $p>0$, follows from the area integral estimates for elliptic systems [DKPV], and the second by the solvability of the $L^{p}$ Dirichlet problem. We refer the reader to [V2, p870-871] for details in the case of Laplace's equation.

We now use integration by parts to obtain

$$
\begin{aligned}
\int_{\partial \Omega} \frac{\partial \mathbf{u}}{\partial \nu} \cdot \mathbf{g} d \sigma & =\int_{\partial \Omega} \mathbf{u} \cdot \frac{\partial \mathbf{v}}{\partial \nu} d \sigma=\int_{\partial \Omega} \mathbf{u} \cdot \frac{\partial \mathbf{w}}{\partial \nu} d \sigma \\
& =(2-n) \int_{\partial \Omega} \mathbf{u} \cdot \frac{\partial \mathbf{H}}{\partial \nu} d \sigma-\int_{\partial \Omega} \frac{\partial u^{r}}{\partial t_{i \ell}} a_{i j}^{r s} \frac{\partial H^{s}}{\partial x_{j}} x_{\ell} d \sigma,
\end{aligned}
$$

where $\frac{\partial}{\partial t_{i \ell}}=n_{i} \frac{\partial}{\partial x_{\ell}}-n_{\ell} \frac{\partial}{\partial x_{i}}$ is a tangential derivative. We remark that the integration by parts used in (4.5) can be justified by an approximation argument, using estimate (4.4) and the assumption that $\nabla \mathbf{u}$ has nontangential limits on $\partial \Omega$ and $(\nabla \mathbf{u})^{*} \in L^{p^{\prime}}$. It follows from (4.5) and Hölder's inequality that

$$
\left|\int_{\partial \Omega} \frac{\partial \mathbf{u}}{\partial \nu} \cdot \mathbf{g} d \sigma\right| \leq C\left\|\nabla_{t} \mathbf{u}\right\|_{p^{\prime}}\|\mathbf{g}\|_{p}
$$

where (4.4) and Poincaré's inequality are also used. The desired estimate then follows by duality.

We now give the proof of Theorem 1.4.

Proof of Theorem 1.4. Let $\Gamma(x)$ denote the matrix fundamental solution for the operator $\mathcal{L}$ on $\mathbb{R}^{n}$ with pole at the origin. Consider the single layer potential

$$
\mathcal{S}(\mathbf{g})(x)=\int_{\partial \Omega} \Gamma(x-y) \mathbf{g}(y) d \sigma .
$$

For the existence as well as the estimate $\left\|(\nabla \mathbf{u})^{*}\right\|_{p} \leq C\left\|\nabla_{t} \mathbf{u}\right\|_{p}$, it suffices to show that there exists $\varepsilon>0$ such that $\mathcal{S}: L^{p}(\partial \Omega) \rightarrow L_{1}^{p}(\partial \Omega)$ is invertible for $\frac{2(n-1)}{n+1}-\varepsilon<p \leq 2$. To this end, we fix $P_{0} \in \partial \Omega$ and assume that

$$
B\left(P_{0}, r_{0}\right) \cap \Omega=B\left(P_{0}, r_{0}\right) \cap\left\{\left(x^{\prime}, x_{n}\right) \in \mathbb{R}^{n}: x_{n}>\psi\left(x^{\prime}\right)\right\}
$$

where $\psi: \mathbb{R}^{n-1} \rightarrow \mathbb{R}$ is Lipschitz continuous. We may assume that $P_{0}=0$. Let

$$
\Omega_{r}=\left\{\left(x^{\prime}, x_{n}\right) \in \mathbb{R}^{n}:\left|x_{1}\right|<r, \ldots,\left|x_{n-1}\right|<r, \psi\left(x^{\prime}\right)<x_{n}<B r\right\}
$$

where the constant $B=B\left(n,\|\nabla \psi\|_{\infty}\right)>0$ is chosen so that $\Omega_{r}$ is a starshaped Lipschitz domain for any $r>0$. Note that, by Theorem 1.3, there exists $\delta>0$ 
depending only on $n, m, \mu$ and $\|\nabla \psi\|_{\infty}$, such that the $L^{q}$ Dirichlet problem for the operator $\mathcal{L}$ on $\Omega_{r}$ is uniquely solvable for any $r>0$ and $2 \leq q<q_{0}=$ $\frac{2(n-1)}{n-3}+\delta$.

Let $\mathbf{g} \in L^{p}(\partial \Omega)$ and $\mathbf{v}=\mathcal{S}(\mathbf{g})$ in $\mathbb{R}^{n}$. Suppose $2 \leq p^{\prime}<q_{0}$. We may apply Lemma 4.2 to $\mathbf{v}$ on $\Omega_{r}$ for $0<r<c r_{0}$. This gives

$$
\int_{\Delta_{r}}\left|\frac{\partial \mathbf{v}}{\partial \nu}\right|^{p} d \sigma \leq C \int_{\partial \Omega_{s r}}\left|\nabla_{t} \mathbf{v}\right|^{p} d \sigma \leq C \int_{\partial \Omega}\left|\nabla_{t} \mathbf{v}\right|^{p} d \sigma+C \int_{\Omega_{\Omega} \cap \Omega_{s r}}|\nabla \mathbf{v}|^{p} d \sigma
$$

where $s \in(1,2)$. By integrating (4.10) in $s$ and covering $\partial \Omega$ with a finite number of coordinate patches, we obtain

$$
\begin{aligned}
\int_{\partial \Omega}\left|\frac{\partial \mathbf{v}}{\partial \nu}\right|^{p} d \sigma & \leq C \int_{\partial \Omega}\left|\nabla_{t} \mathbf{v}\right|^{p} d \sigma+C \int_{\widetilde{\Omega}}|\nabla \mathbf{v}|^{p} d x \\
& \leq C \int_{\partial \Omega}\left|\nabla_{t} \mathbf{v}\right|^{p} d \sigma+\gamma \int_{\partial \Omega}\left|(\nabla \mathbf{v})^{*}\right|^{p} d \sigma+C_{\gamma} \sup _{K}|\mathbf{v}|^{p},
\end{aligned}
$$

where $\widetilde{\Omega}=\left\{x \in \Omega: \operatorname{dist}(x, \partial \Omega)<r_{0}\right\}$, and $K$ is a compact set in $\Omega$. To estimate $\mathbf{v}$ on $K$, we choose $\bar{q}<2$ so that the $L^{\bar{q}}$ Dirichlet problem is uniquely solvable. It follows that

$$
\sup _{K}|\mathbf{v}| \leq C\left\|(\mathbf{v})^{*}\right\|_{\bar{q}} \leq C\|\mathbf{v}\|_{\bar{q}} \leq C\left\{\left\|\nabla_{t} \mathbf{v}\right\|_{\bar{p}}+\|\mathbf{v}\|_{\bar{p}}\right\},
$$

where $\frac{1}{\bar{q}}=\frac{1}{\bar{p}}-\frac{1}{n-1}$, and we used the Sobolev imbedding in the last inequality. Note that $\bar{q}<2$ implies $\bar{p}<2(n-1) /(n+1)$. We may assume that $\bar{p}^{\prime}<$ $2(n-1) /(n-3)+\delta$. Thus, if $\bar{p} \leq p \leq 2$, we obtain

$$
\int_{\partial \Omega}\left|\frac{\partial \mathbf{v}}{\partial \nu}\right|^{p} d \sigma \leq C \int_{\partial \Omega}\left|\nabla_{t} \mathbf{v}\right|^{p} d \sigma+C \int_{\partial \Omega}|\mathbf{v}|^{p} d \sigma+\gamma C \int_{\partial \Omega}|\mathbf{g}|^{p} d \sigma
$$

where we have used the estimate $\left\|(\nabla \mathbf{v})^{*}\right\|_{p} \leq C\|\mathbf{g}\|_{p}$ (see e.g. [V1] or [K1]). By the same argument, estimate (4.13) also holds for the exterior domain $\mathbb{R}^{n} \backslash \bar{\Omega}$. Thus, by the jump relation $\mathbf{g}=\frac{\partial \mathbf{v}_{+}}{\partial \nu}-\frac{\partial \mathbf{v}_{-}}{\partial \nu}$ where \pm indicate the limits taken from $\Omega$ and $\mathbb{R}^{n} \backslash \bar{\Omega}$ respectively, we obtain

$$
\|\mathbf{g}\|_{p} \leq\left\|\frac{\partial \mathbf{v}_{+}}{\partial \nu}\right\|_{p}+\left\|\frac{\partial \mathbf{v}_{-}}{\partial \nu}\right\|_{p} \leq C\left\{\left\|\nabla \mathbf{v}_{t}\right\|_{p}+\|\mathbf{v}\|_{p}+\gamma\|\mathbf{g}\|_{p}\right\},
$$

for $\bar{p} \leq p \leq 2$. Choose $\gamma>0$ small so that $\gamma C<1 / 2$. This gives

$$
\|\mathbf{g}\|_{p} \leq C\|\mathcal{S}(\mathbf{g})\|_{L_{1}^{p}(\partial \Omega)} \text {. }
$$

Estimate (4.15) implies that the operator $\mathcal{S}: L^{p}(\partial \Omega) \rightarrow L_{1}^{p}(\partial \Omega)$ is one-to-one and has a closed range. Note that the range is also dense, since $\mathcal{S}: L^{2}(\partial \Omega) \rightarrow$ $L_{1}^{2}(\partial \Omega)$ is invertible $[\mathrm{G}]$. We conclude that $\mathcal{S}: L^{p}(\partial \Omega) \rightarrow L_{1}^{p}(\partial \Omega)$ is invertible for $\bar{p} \leq p \leq 2$.

Finally, to show the uniqueness, we suppose that $\mathcal{L} \mathbf{u}=\mathbf{0}$ in $\Omega, \mathbf{u}=0$ on $\partial \Omega$ and $(\nabla \mathbf{u})^{*} \in L^{p}$ for some $p>2(n-1) /(n+1)-\varepsilon$. It follows from $(2.10)$ that $(\mathbf{u})^{*} \in L^{q}$, where $\frac{1}{q}=\frac{1}{p}-\frac{1}{n-1}$. Note that if $\varepsilon>0$ is small, $q>2-\bar{\varepsilon}$. Thus the uniqueness in Theorem 1.4 follows from the uniqueness of the $L^{q}$ Dirichlet problem for $q>2-\bar{\varepsilon}[\mathrm{G}]$. 


\section{References}

[CP] L.A. Caffarelli and I. Peral, On $W^{1, p}$ estimates for elliptic equations in divergence form, Comm. Pure App. Math. 51 (1998), 1-21.

[D1] B. Dahlberg, On estimates for harmonic measure, Arch. Rat. Mech. Anal. 65 (1977), 273-288.

[D2] - On the Poisson integral for Lipschitz and $C^{1}$ domains, Studia Math. 66 (1979), 13-24.

[DK1] B. Dahlberg and C. Kenig, Hardy spaces and the Neumann problem in $L^{p}$ for Laplace's equation in Lipschitz domains, Ann. of Math. 125 (1987), 437-466.

[DK2] $L^{p}$ estimates for the three-dimensional systems of elastostatics on Lipschitz domains, Lecture Notes in Pure and Appl. Math. 122 (1990), 621-634.

[DKPV] B. Dahlberg, C. Kenig, J. Pipher, and G. Verchota, Area integral estimates for higher order elliptic equations and systems, Ann. Inst. Fourier (Grenoble) 47 (1997), 14251461.

[DKV1] B. Dahlberg, C. Kenig, and G. Verchota, The Dirichlet problem for the biharmonic equation in a Lipschitz domain, Ann. Inst. Fourier (Grenoble) 36 (1986), 109-135.

[DKV2] _ Boundary value problems for the systems of elastostatics in Lipschitz domains, Duke Math. J. 57 (1988), 795-818.

[F] E. Fabes, Layer potential methods for boundary value problems on Lipschitz domains, Lecture Notes in Math. 1344 (1988), 55-80.

[FKV] E. Fabes, C. Kenig, and G. Verchota, Boundary value problems for the Stokes system on Lipschitz domains, Duke Math. J. 57 (1988), 769-793.

[G] W. Gao, Boundary value problems on Lipschitz domains for general elliptic systems, J. Funct. Anal. (1991), 377-399.

[Gi] M. Giaquinta, Multiple Integrals in the Calculus of Variations and Nonlinear Elliptic Systems, Annals of Math. Studies 105, Princeton Univ. Press, 1983.

[JK] D. Jerison and C. Kenig, The Neumann problem in Lipschitz domains, Bull. Amer. Math. Soc. 4 (1981), 203-207.

[K1] C. Kenig, Elliptic boundary value problems on Lipschitz domains, Beijing Lectures in Harmonic Analysis, Ann. of Math. Studies 112 (1986), 131-183.

[K2] Harmonic Analysis Techniques for Second Order Elliptic Boundary Value Problems, CBMS Regional Conference Series in Math., vol. 83, AMS, Providence, RI, 1994.

[MM] D. Mitrea and M. Mitrea,, General second order, strongly elliptic systems in low dimensional nonsmooth manifold, Contemporary Math. 277 (2001), 61-86.

[PV1] J. Pipher and G. Verchota, The Dirichlet problem in $L^{p}$ for the biharmonic equation on Lipschitz domains, Amer. J. Math. 114 (1992), 923-972.

[PV2] - A maximum principle for biharmonic functions in Lipschitz and $C^{1}$ domains, Commen. Math. Helv. 68 (1993), 385-414.

[PV3] Dilation invariant estimates and the boundary Garding inequality for higher order elliptic operators, Ann. of Math. (2) 142 (1995), 1-38.

[PV4] Maximum principle for the polyharmonic equation on Lipschitz domains, Potential Analysis 4 (1995), 615-636.

[S1] Z. Shen, Resolvent estimates in $L^{p}$ for elliptic systems in Lipschitz domains, J. Funct. Anal. 133 (1995), 224-251.

[S2] - A note on the Dirichlet problem for the Stokes system in Lipschitz domains, Proc. Amer. Math. Soc. 123 (1995), 801-811.

[S3] _ Boundary value problems in Morrey spaces for elliptic systems on Lipschitz domains, Amer. J. Math. 125 (2003), 1079-1115.

[S4] - Weighted estimates for elliptic systems in Lipschitz domains, to appear in Indiana Univ. Math. J.. 
[S5] B Bounds of Riesz transforms on $L^{p}$ spaces for second order elliptic operators, Ann. Inst. Fourier (Grenoble) 55 (2005), no. 1, 173-197.

[V1] G. Verchota, Layer potentials and regularity for the Dirichlet problem for Laplace's equation, J. Funct. Anal. 59 (1984), 572-611.

[V2] $\quad$ The Dirichlet problem for the biharmonic equation in $C^{1}$ domains, Indiana Univ. Math. J. 36 (1987), 867-895.

[V3] The Dirichlet problem for the polyharmonic equation in Lipschitz domains, Indiana Univ. Math. J. 39 (1990), 671-702.

Department of Mathematics, University of Kentucky, Lexington, KY 40506.

E-mail address: shenz@ms.uky.edu 